\title{
Phenotype of distinct primary sensory afferent subpopulations and caspase-3 expression following axotomy
}

\author{
Adam J. Reid · Cristina Mantovani · \\ Susan G. Shawcross • Giorgio Terenghi • Mikael Wiberg
}

Accepted: 27 May 2011 / Published online: 15 June 2011

(C) Springer-Verlag 2011

\begin{abstract}
Specific sensory neuronal subpopulations show contrasting responses to peripheral nerve injury, as shown by the axotomy-induced death of many cutaneous sensory neurons whilst muscular sensory afferents survive an identical insult. We used a novel combination of retrograde neuronal tracing with immunohistochemistry and laser microdissection techniques, in order to describe the neurochemistry of medial gastrocnemius (muscular sensory afferents) and sural (cutaneous sensory afferents) branches of the rat sciatic nerve and relate this to the pro-apoptotic caspase-3 gene expression following nerve transection. Our results demonstrated distinctions in medial gastrocnemius and sural neuron populations with the most striking difference in the respective proportions of isolectin B4 (IB4) staining neurons ( $3.7 \mathrm{~V} 32.8 \%$ ). The mean neuronal area of the medial gastrocnemius (MG) neurons was larger than that of the sural (SUR) neurons $\left(1,070.8 \mathrm{~V} 646.2 \mu \mathrm{m}^{2}\right)$ and each phenotypic group was significantly smaller in sural neurons than in MG neurons. At 1 week post-axotomy, MG neurons markedly downregulated caspase-3, whilst SUR neurons upregulated caspase- 3 gene expression; this may be attributable to the differing IB4-positive composition of the subpopulations. These findings provide further clarification in the understanding of two distinct neuronal populations used increasingly in nerve injury models.
\end{abstract}

A. J. Reid $(\bowtie) \cdot$ C. Mantovani $\cdot$ S. G. Shawcross $\cdot$ G. Terenghi Blond McIndoe Research Laboratories, Tissue Injury and Repair Group, University of Manchester, Stopford Building, Oxford Road, Manchester M13 9PT, UK

e-mail: adam.reid@manchester.ac.uk

A. J. Reid · M. Wiberg

Departments of Anatomy and Hand and Plastic Surgery,

Umeå University, Umeå, Sweden
Keywords Rat · Peripheral nerve - Dorsal root ganglion Neuronal phenotype $\cdot$ Neuronal apoptosis $\cdot$ Caspase-3

\section{Introduction}

Adult dorsal root ganglia (DRG) comprise a heterogenous pool of sensory neurons that can be divided into subpopulations according to several parameters including neuronal size, neurochemistry, trophic requirements and sensory modality (Lawson 2005). Recently, a differential response to peripheral nerve transection has been recognised with DRG neuronal loss which is earlier and more severe in B-cells (small, dark cells with unmyelinated axons) than A-cells (large, light cells with myelinated axons) (Tandrup et al. 2000). Further to this, contrasting responses to peripheral nerve injury have been demonstrated in functionally distinct sensory subpopulations. Using a rat model in which sural and medial gastrocnemius branches of the sciatic nerve are selectively axotomised, it has been shown that cutaneous sensory afferents die in great numbers following axotomy, whereas muscular sensory afferents survive an identical insult ( $\mathrm{Hu}$ and McLachlan 2003; Welin et al. 2008).

Whilst previous studies have determined a correlation between the neurochemical phenotype of DRG neurons and their function (Lawson et al. 2002; McLachlan and Janig 1983; Molander et al. 1987; O'Brien et al. 1989), it is clear that peripheral nerve injury induces neurochemical plasticity in DRG neurons (Bradbury et al. 1998; McMahon and Moore 1988). Yet to be established is the relationship between the neurochemistry of axotomised populations and their propensity to apoptotic death.

DRG neuronal phenotype can be distinguished by immunocytochemical markers of different size classes. Small 
unmyelinated afferents are comprised of two populations differentiated by their potential to synthesise neuropeptides-peptidergic afferents are predominantly of nociceptive function and identified by their immunoreactivity for calcitonin gene-related peptide (CGRP), whilst the exclusively nociceptive non-peptidergic afferents are identified by their binding of Griffonia simplicifolia isolectin B4 (IB4). Myelinated primary afferents, which innervate peripheral mechanoreceptors and proprioceptors, are identified by their binding of the high molecular weight neurofilament (NF-H) which recognises a $200 \mathrm{kD}$ neurofilament protein (Lawson 2005).

Axotomy-induced sensory neuronal death is recognised to be mediated through apoptosis comprising a cascade of pathways culminating in caspase- 3 activation which effects the death-inducing proteolytic action on cells (Groves et al. 1997; Janicke et al. 1998). Following peripheral axotomy morphologically apoptotic DRG neurons demonstrate immunoreactivity for activated caspase- 3 protein (Kuo et al. 2005), and the proportion of IB4-positive neurons decreases in the axotomised DRG (Bradbury et al. 1998; McMahon and Moore 1988). Therefore, we were keen to investigate the relationship between neurochemical phenotype and apoptotic expression in functionally differing DRG subpopulations.

Here, we present data characterising medial gastrocnemius and sural neuron neurochemical phenotype and neuronal profile size. In a parallel experiment, a novel combination of fluorescent retrograde labelling, laser microdissection and real-time PCR is utilised to examine our results in the context of caspase- 3 gene expression in these functional subpopulations following selective axotomy.

\section{Materials and methods}

\section{Retrograde neuronal labelling}

Experiments were performed on young adult male Wistar rats $(220-250 \mathrm{~g})$ anaesthetised with isofluorane. The animal care and experimental procedures were performed in accordance with the terms of the Animals (Scientific Procedures) Act 1986 and the number of animals used was kept to a minimum. Animals underwent left sided unilateral medial gastrocnemius (MG, sensory neurons projecting from muscle) nerve $(n=8)$ or sural (SUR, sensory neurons projecting from skin) nerve $(n=8)$ division at the same level in the popliteal fossa of the left hind limb. The proximal nerve end was introduced into a small polyethylene cap containing $2 \mu \mathrm{L}$ of Fast Blue (FB, 2\% aqueous solution, EMS-Chemie $\mathrm{GmbH}$, Germany) and sealed with a mixture of silicone grease and Vaseline to prevent leakage. Animals were kept warm on a heated blanket and regular observations were made to ensure a satisfactory anaesthetic plane. The cap was removed $2 \mathrm{~h}$ later; proximal nerve was rinsed in saline, wrapped in Spongostan absorbable gelatin sponge (Johnson and Johnson Medical, UK) to prevent dye leakage and directed away from the distal nerve ending to prevent regeneration. The wound was closed in layers.

Control undamaged neuronal labelling was required for baseline caspase- 3 gene expression values. For control undamaged MG neuron labelling, $4 \mu \mathrm{L}$ Fast Blue tracer was injected into the left medial gastrocnemius muscle $(n=5)$ at four different sites using a $10 \mu \mathrm{L}$ Hamilton syringe (Hamilton, Switzerland). The tracer was allowed to dissipate through the muscle before the needle was withdrawn and the muscle carefully washed and dried. Control SUR neuronal labelling was performed by injecting $2 \mu \mathrm{L}$ Fast Blue tracer into the sural nerve $(n=5)$ using a fine glass microelectrode. These techniques labelled considerably fewer neurons than the axotomy technique above; however, at least 100 neurons were labelled in each animal enabling sufficient neuron capture by laser microdissection for gene expression analysis.

Two methods were used to test for the presence of nonspecific labelling. First, the contralateral DRG were examined for Fast Blue staining. Second, in a separate animal the axotomised sural nerve was divided and capped proximal to the site of Fast Blue administration to prevent dye uptake. These control experiments confirmed that there was no leakage of the dye.

Experimental groups

Phenotyping groups at 4 days after nerve transection, three animals from both MG and SUR axotomy studies were deeply anaesthetised with isofluorane and perfused transcardially with $0.9 \%$ heparinised saline $(0.9 \% \mathrm{NaCl}$, 50 units $/ \mathrm{ml}$ heparin) followed by $4 \%$ paraformaldehyde in $0.1 \mathrm{M}$ phosphate buffer solution (PBS). Ipsilateral and contralateral L4 and L5 DRG were harvested and post-fixed in $4 \%$ paraformaldehyde before being frozen in OCT embedding medium (VWR, UK) and stored at $-40^{\circ} \mathrm{C}$.

Caspase-3 mRNA expression groups at 7 days after nerve transection, five animals from both MG and SUR axotomy and the undamaged MG and SUR neuron labelled groups were killed by cervical dislocation. Ipsilateral and contralateral L4 and L5 DRG were harvested carefully but rapidly and flash frozen in OCT embedding medium (VWR, UK) and stored at $-40^{\circ} \mathrm{C}$.

\section{Immunohistochemistry}

Phenotyping groups serial $14 \mu \mathrm{m}$ cryosections of DRG were cut longitudinally using Bright (UK) 5040 microtome, 
mounted onto Superfrost Plus slides (VWR, UK) and airdried. Slides were washed five times in PBS, and then incubated for $1.5 \mathrm{~h}$ in $5 \%$ normal donkey serum (Sigma, UK), $0.2 \%$ Triton-X-100 in PBS. Sections were then incubated in primary antibody solution overnight at $4^{\circ} \mathrm{C}$. The primary antibodies used in this study were polyclonal rabbit NF-H (1:400, Chemicon), monoclonal mouse CGRP (1:500, Sigma) and FITC-conjugated IB4 from Griffonia simplicifolia $(1 \mathrm{mg} / \mathrm{ml}$, Vector Labs, UK). Sections were washed in PBS then incubated for $1.5 \mathrm{~h}$ in fluorescein isothiocyanate (FITC) and/or cyanine 3 (Cy3)-conjugated donkey anti-rabbit or anti-mouse as appropriate (1:100; Jackson ImmunoResearch). Sections were mounted in Vectorshield (Vector Labs).

In each rat, retrogradely-labelled L4/5 DRG were doublestained with two out of three antibodies (CGRP, IB4 or NF-H) which enabled assessment of each antibody phenotype across two animals in both MG and SUR nerve injury groups. Immunofluorescence was visualised using an Olympus BX60 inverted fluorescence microscope and images acquired using a monochrome camera (Evolution QEi, MediaCybernetics, Bethesda, USA) and processed using Image-Pro Plus Imaging software (Media Cybernetics).

In the medial gastrocnemius nerve injury group the entire FB-labelled neuronal population of over 200 cells was included for counting. A neuron was counted when the nucleus was visible and adjacent sections were examined to limit double counting of split cells. Every MG neuron was then examined for positive staining with the phenotyping antibodies. Due to the large number of sural neurons, this population was sampled with every fifth section examined for FB-labelled neurons which again were only counted if the nucleus was visible. These neurons were also examined for co-staining with the phenotyping antibodies and over 200 FB neuronal profiles were counted in order to have a similar number of cells as the MG population. The Image Pro Plus software was also used to measure the maximal area of each neuronal profile counted.

\section{Laser microdissection}

Caspase-3 mRNA expression groups serial $10 \mu \mathrm{m}$ cryosections of DRG were cut using Bright (UK) 5040 microtome and mounted onto RNase-free UV light-treated membrane slides: $1 \mathrm{~mm}$ polyethylene-tetraphthalate (PET) membrane, PALM (Zeiss, Germany). Slides were air-dried in the cryostat before being fixed in ice-cold methacarn (eight parts methanol, one part glacial acetic acid) for $10 \mathrm{~min}$, dipped into ice-cold RNase-free PBS to remove excess OCT, and then in serial ethanol 70/96/100\% for $2 \mathrm{~min} /$ $2 \mathrm{~min} / 3 \mathrm{~min}$, respectively, for tissue dehydration.

Laser capture microdissection was performed on PALM Microlaser Technologies microbeam microdissection system (Zeiss, Germany). Retrogradely-labelled fluorescent neurons were visualised, marked under fluorescence illumination (UV filter, $350 \mathrm{~nm}$ excitation) and cut. The PET membrane of the slide is cut simultaneously and provides a 'back-bone' to facilitate laser pressure catapulting-a precisely aimed but defocused high-energy laser beam to catapult the area against gravity into the collecting microfuge tube. Individual neurons were catapulted contact-free into a PALM AdhesiveCap with a total of 100 cells captured for each animal. This procedure allows collection of material in a contact-free manner, minimising the risk of contamination which is particularly important in sensitive downstream applications.

\section{Quantitative RT-PCR}

Laser-captured samples were submerged in $350 \mu \mathrm{L}$ lysis buffer (RLT buffer, QIAGEN, Germany) and 3.5 $\mu \mathrm{L} \beta$-mercapotethanol (Sigma-Aldrich, UK) was added alongside 20 ng carrier RNA before homogenisation. The samples were incubated upside down for $30 \mathrm{~min}$, then vortexed thoroughly. RNeasy (Qiagen) Micro protocol for isolation of total RNA from microdissected cryosections was undertaken including the optional DNase step. The purified RNA was eluted in $14 \mu \mathrm{L}$ RNase-free water.

All total RNA samples were converted to cDNA using a First-Strand cDNA Synthesis Kit (Superarray, USA). qRT-PCR was performed with a Rotor-Gene $6000+$ HRM (Corbett Life Science, Australia) using SYBR ${ }^{\circledR}$ Green fluorescence master-mix (SABiosciences Corporation, MD, USA) and analysed using Rotor-Gene 6000 Series Software version 1.7.61 (Corbett Life Science, Australia). Primers were pre-designed by Superarray-housekeeping gene HPRT (Genbank Accession No. NM012583, Catalogue No. PPR44247E) and caspase-3 (Acc. No. NM012922, Cat. No. PPR06384A). All reactions had been optimised to work under the same conditions-initial denaturation/HotStart DNA Polymerase activation: $95^{\circ} \mathrm{C}$ for $15 \mathrm{~min}$; PCR cycles: $95^{\circ} \mathrm{C}$ for $30 \mathrm{~s}, 55^{\circ} \mathrm{C}$ for $30 \mathrm{~s}$, and $72^{\circ} \mathrm{C}$ for $30 \mathrm{~s}$ repeated for 40 cycles. One PCR run was performed for both genes and contained a standard curve, generated from serial dilutions of testicular tissue cDNA over four orders of magnitude; all experimental samples were assayed in triplicate. A negative control assay was always included where cDNA template was replaced with RNase-free water.

Data from a reaction was excluded only if its threshold cycle $C(t)$, differed by more than one cycle compared with the other two reactions in the triplicate run. From the standard curves described above, the $\mathrm{C}(\mathrm{t})$ values were used to calculate mRNA levels (arbitrary units) in each sample. For every animal, the expression level of caspase-3 was normalised to that of the housekeeping gene, HPRT. Confirmation of the amplified products was established by performing a 
melting curve analysis: $95^{\circ} \mathrm{C}$ for $1 \mathrm{~min}, 65^{\circ} \mathrm{C}$ for $2 \mathrm{~min}$, then $65-95^{\circ} \mathrm{C}$, reading every $0.2^{\circ} \mathrm{C}$, holding for $1 \mathrm{~s}$ between reads.

\section{Statistics}

GraphPad Prism 4@ software (GraphPad Software, San Diego, CA, USA) was used to calculate statistics and an unpaired student $t$ test was used to compare neuronal crosssectional distances in phenotypic groups across MG and SUR subpopulations. One-way ANOVA test was used to determine differences in gene expression between the groups. Post-hoc Bonferroni's Multiple Comparison test was used to compare axotomised with non-axotomised MG and sural neurons, respectively. All data were expressed as mean \pm SEM. A value of $P<0.05$ was considered to be statistically significant.

\section{Results}

In both MG and sural labelled populations, each phenotyping antibody (CGRP, IB4 and NF-H) stained L4/5 DRG of two animals in order that a distinct double-staining combination was used in each of three animals. DRG sections with FB-labelled MG neurons were shown to co-stain with NF-H (green) and CGRP (red) (Fig. 1). Numerous NF-H and CGRP-positive neurons were observed with few MG neurons and in Fig. 1 only one of each phenotype is seen to co-localise with FB. The FB-labelled CGRP-positive neuron is identified by the purple (red plus blue) colour and the FB-labelled NF-H positive neuron is identified by the blue plus green colour. FB-labelled sural neurons co- localised with CGRP (red) and IB4 (green) (Fig. 2). The FB-labelled

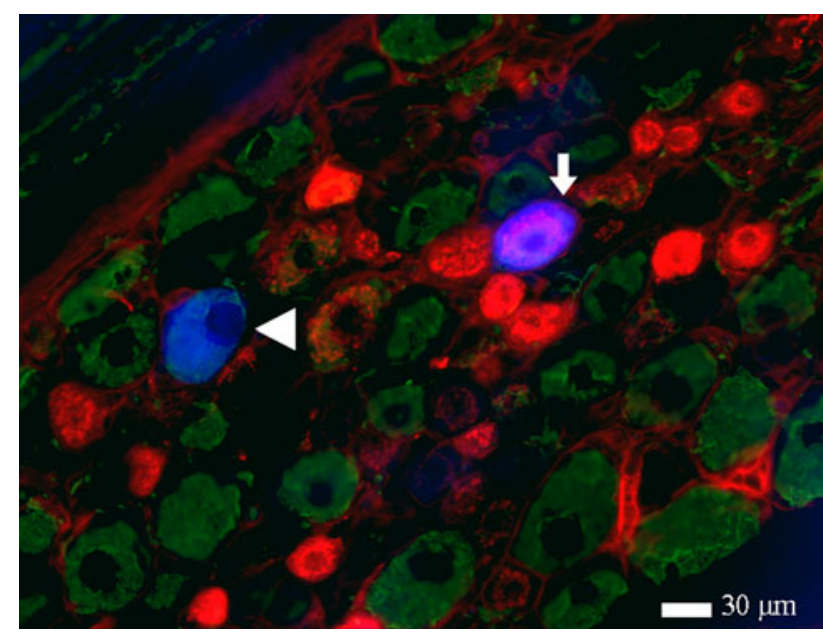

Fig. 1 FB-labelled MG neurons co-localised with NF-H (green) and CGRP (red). Arrow shows FB-labelled CGRP-positive MG neuron as purple colour. Arrowhead shows FB-labelled NF-H positive MG neuron as blue plus green colour. Scale bar $30 \mu \mathrm{m}$. 20× magnification

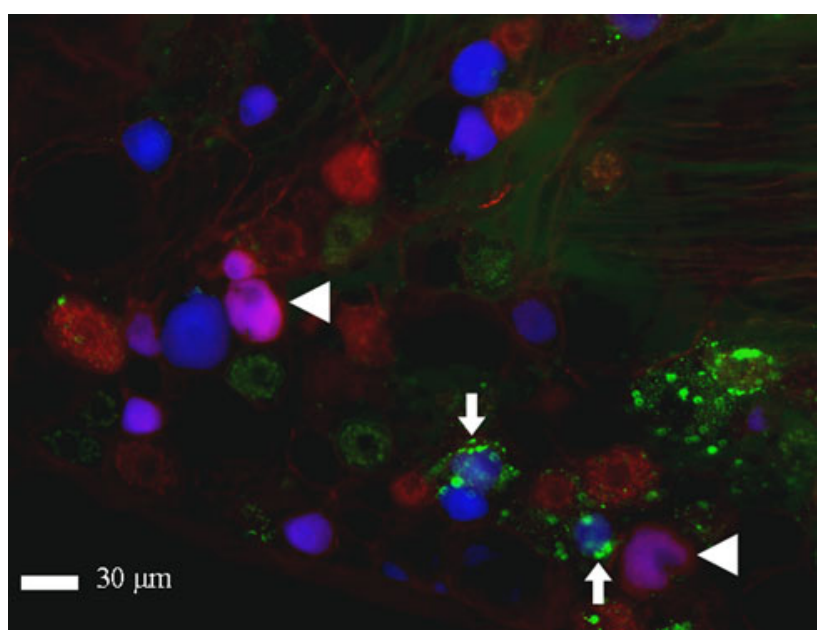

Fig. 2 FB-labelled sural neurons co-localised with CGRP (red) and IB4 (green). Arrowheads show FB-labelled CGRP-positive sural neuron as purple colour. Arrows show FB-labelled IB4 positive sural neuron as blue plus granular green staining. Scale bar $30 \mu \mathrm{m} .20 \times$ magnification

CGRP-positive neurons are identified again by the purple colour, whilst the FB-labelled IB4 positive neurons are identified by the blue plus green granular staining. FBlabelled neurons are clearly more numerous in Fig. 2 than in Fig. 1 demonstrating the contrast in neuronal population size. In the L4/5 DRG, there are an estimated 3,500 sural neurons compared with only $220 \mathrm{MG}$ neurons (Welin et al. 2008). It is also evident from these figures that the sural neurons are smaller in size than the MG neurons.

\section{Neurochemical characterisation of MG and SUR neurons}

The mean number of FB profiles and phenotype co-localisation across three animals was calculated (Table 1). In FB-labelled MG afferents, a large proportion showed co-localisation with NF-H immunostaining (49.3\%) and CGRP immunostaining (40.6\%), whilst only a small proportion showed co-localisation with IB4 immunostaining (3.7\%). A small percentage of the FB-labelled MG neurons co-localised with two antibodies-CGRP and NF-H (4.1\%), CGRP and IB4 (0.9\%) and NF-H and IB4 (1.4\%). FB-labelled sural afferents demonstrated a more even share of phenotyping antibody co-localisation with NF-H (30.7\%), CGRP (27.9\%) and IB4 immunostaining (32.8\%). There was also a small percentage of FB-labelled sural neurons that co-localised with two antibodies-CGRP and NF-H (3.3\%), CGRP and IB4 (4.5\%) and NF-H and IB4 (0.8\%).

Profile size of MG and SUR neurons

Maximum neuronal area was measured in every MG and sural profile counted (Fig. 3). The mean area of the MG 
Table 1 Mean numbers and percentage of MG and SUR neurons that co-stain with phenotypic antibodies

\begin{tabular}{|c|c|c|c|c|c|c|c|}
\hline MG & Antibody & $\begin{array}{l}\text { Mean number of } \\
\text { neurons } \pm \text { SEM }\end{array}$ & $\%$ & SUR & Antibody & $\begin{array}{l}\text { Mean number of } \\
\text { neurons } \pm \text { SEM }\end{array}$ & $\%$ \\
\hline & $\operatorname{CGRP}(N=2)$ & $89 \pm 4.0$ & 40.6 & & $\operatorname{CGRP}(N=2)$ & $68 \pm 2.5$ & 27.9 \\
\hline & IB4 $(N=2)$ & $8 \pm 1.0$ & 3.7 & & $\mathrm{IB} 4(N=2)$ & $80 \pm 2.5$ & 32.8 \\
\hline & NF-H $(N=2)$ & $108 \pm 6.5$ & 49.3 & & NF-H $(N=2)$ & $75 \pm 4.0$ & 30.7 \\
\hline & CGRP + IB4 & 2 & 0.9 & & CGRP + IB4 & 11 & 4.5 \\
\hline & $\mathrm{CGRP}+\mathrm{NF}-\mathrm{H}$ & 9 & 4.1 & & $\mathrm{CGRP}+\mathrm{NF}-\mathrm{H}$ & 8 & 3.3 \\
\hline & $\mathrm{IB} 4+\mathrm{NF}-\mathrm{H}$ & 3 & 1.4 & & $\mathrm{IB} 4+\mathrm{NF}-\mathrm{H}$ & 2 & 0.8 \\
\hline Total & & 219 & 100 & Total & & 244 & 100 \\
\hline
\end{tabular}

In both MG and sural labelled populations, each phenotyping antibody stained L4/5 DRG of two animals in order that a distinct double-staining combination was used in each of three animals. The mean number of FB-labelled neurons staining with each individual antibody (across two animals) and the true number of double-staining FB-labelled neurons (in one animal) are pooled to represent the phenotypic makeup of MG and sural populations

$M G$ Animal with FB-labelled MG neurons. SUR Animal with FB-labelled sural neurons

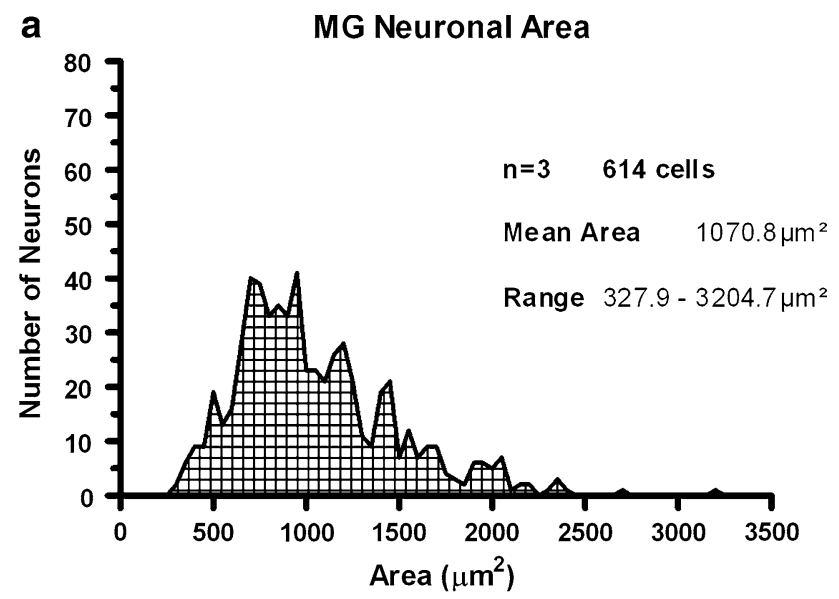

b

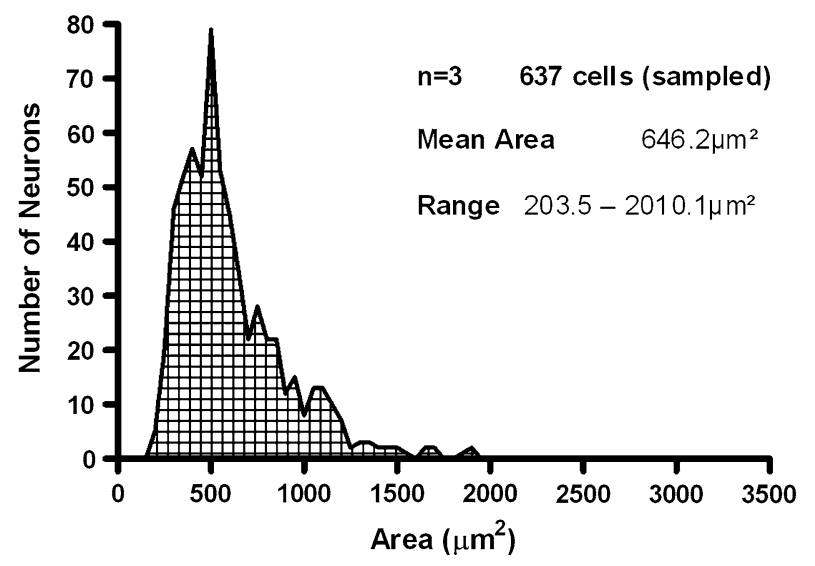

Fig. 3 a MG neuronal area distribution. The neuronal area distribution of every FB-labelled MG neuron counted inthree animals shows a normal distribution with a wide range and a mean neuronal area of $1,070.8 \pm 17.2 \mu \mathrm{m}^{2}$. b Sural neuron area distribution. The neuronal area distribution of every FB-labelled sural neuron counted in three animals shows a skew towards neurons of smaller size and a mean area of $646.2 \pm 11.6 \mu \mathrm{m}^{2}$
Table 2 Mean neuronal area and range of MG and sural neurons subdivided into phenotypic groups

\begin{tabular}{lcll}
\hline & $\begin{array}{l}\text { Number of } \\
\text { neurons }\end{array}$ & $\begin{array}{l}\text { Mean neuronal } \\
\text { area }\left(\mu \mathrm{m}^{2}\right)\end{array}$ & Range $\left(\mu \mathrm{m}^{2}\right)$ \\
\hline MG neurons & & & \\
CGRP & 178 & $862.8 \pm 20.3$ & $331.1-1,903.2$ \\
IB4 & 16 & $698.7 \pm 40.5$ & $509.2-947.6$ \\
NF-H & 217 & $1,265.6 \pm 30.6$ & $350.0-3,204.7$ \\
Sural neurons & & & \\
CGRP & 137 & $608.2 \pm 22.4$ & $227.6-1,506.7$ \\
IB4 & 159 & $569.0 \pm 25.4$ & $203.5-1,881.0$ \\
NF-H & 150 & $791.5 \pm 26.3$ & $320.6-2,010.1$ \\
\hline
\end{tabular}

The mean neuronal area \pm SEM and the range of neuronal area for MG and sural neurons were calculated in relation to subdivision into phenotypic groups. The mean neuronal area of each phenotypic group was smaller in sural neurons than in MG neurons

MG versus Sural-CGRP $P<0.0001 ;$ NF-H $P<0.0001$

neuron population (Fig. 3a) was $1,070.8 \pm 17.2 \mu \mathrm{m}^{2}$ (range 327.9-3,204.7 $\left.\mu \mathrm{m}^{2}\right)$ whilst the mean area of the sural neuron population (Fig. 3b) was $646.2 \pm 11.6 \mu \mathrm{m}^{2}$ (range 203.5-2,010.1 $\mu \mathrm{m}^{2}$ ). The small neurons of the sural population $\left(<500 \mu \mathrm{m}^{2}\right)$ outnumbered the large $\left(>500 \mu \mathrm{m}^{2}\right)$ by almost 9:1, as seen in Fig. 3 which shows the sural neuronal areas skewed towards neurons of smaller size. This contrasts with the MG neuronal areas which are closer to a normal distribution with a wider range. The mean neuronal areas of MG and sural neurons were calculated in relation to subdivision into phenotypic groups (Table 2). The mean neuronal areas of each phenotypic group were smaller in sural neurons than in MG neurons. The mean neuronal area of CGRP neurons in MG afferents was $862.8 \pm 20.3 \mu \mathrm{m}^{2}$, whilst in sural afferents mean neuronal area was $608.2 \pm 22.4 \mu^{2}(P<0.0001)$; mean neuronal area of IB4 neurons in MG afferents was $698.7 \pm 40.5 \mu \mathrm{m}^{2}$, whilst in 


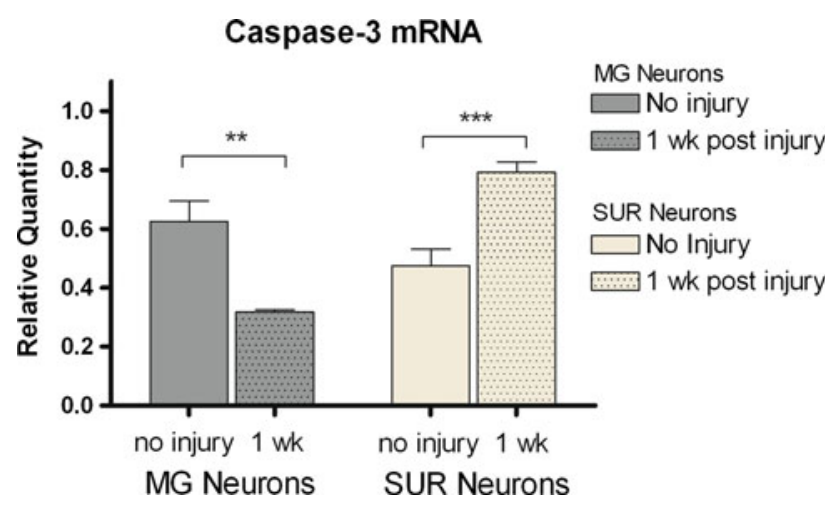

Fig. 4 Relative expression of caspase-3 in MG and SUR neurons qRT-PCR analysis of caspase-3 expression from laser microdissected non-injured and injured medial gastrocnemius neurons (left) and sural neurons (right). The injured neurons were measured at 1 week postaxotomy. Expression values are relative to HPRT expression and are therefore in arbitrary units. Data expressed as mean $\pm \mathrm{SEM}$ $* * P<0.01, * * * P<0.001$

sural afferents mean neuronal area was $569.0 \pm 25.4 \mu \mathrm{m}^{2}$; mean neuronal area of NF-H neurons in MG afferents was $1,265.6 \pm 30.6 \mu \mathrm{m}^{2}$, whilst in sural afferents mean neuronal area was $791.5 \pm 26.3 \mu \mathrm{m}^{2}(P<0.0001)$.

\section{Caspase-3 mRNA expression in MG and SUR neurons}

In response to axotomy, sural and MG neurons have contrasting transcriptional regulation of caspase-3 mRNA (Fig. 4). Caspase- 3 expression was $0.63 \pm 0.07$ in medial gastrocnemius neurons with no injury $(n=5)$ and $0.32 \pm 0.01$ at 1 week post-injury $(n=5$, Bonferroni's post hoc $P<0.01$ ), whilst in sural neurons with no injury the mean relative expression of caspase- 3 was $0.47 \pm 0.06$ $(n=5)$ and $0.79 \pm 0.03$ at 1 week post-injury $(n=5$, $P<0.001)$. Whilst MG neurons markedly downregulate caspase-3, SUR neurons upregulate its gene expression at 1 week post-injury.

\section{Discussion}

Branches of the sciatic nerve are increasingly being used in the study of nerve injury and neuronal survival ( $\mathrm{Hu}$ and McLachlan 2003; Oaklander and Brown 2004; Puigdellivol-Sanchez et al. 2006; Welin et al. 2008). Despite several studies examining the neurochemistry of cutaneous and muscular sensory afferents (Ambalavanar et al. 2003; Lawson et al. 2002; McLachlan and Janig 1983; Molander et al. 1987; O'Brien et al. 1989), those properties of the medial gastrocnemius and sural neurons in the rat have not yet been clearly characterised. In utilising a combination of retrograde neuronal tracing with immunohistochemistry and laser microdissection techniques, we sought to describe the neurochemistry of MG and sural neurons and relate this to the pro-apoptotic caspase- 3 gene expression seen following nerve transection.

Given our methodology of identifying neuronal populations at 4 days post-axotomy, an important consideration is the phenotypic plasticity of neurons following nerve injury. The plasticity of peptide expression has been a recognised phenomenon since Substance $P$ was demonstrated to diminish in the DRG and dorsal horn 7 days after rat sciatic nerve transection (Jessell et al. 1979). More recent studies have observed downgraded expression with reduced CGRP and IB4 positivity in response to nerve injury although NF-H was not significantly altered (Bergman et al. 1999; Hammond et al. 2004). It is difficult, however, to determine the extent to which these results may be due to selective neuronal cell death or phenotypic shift because neuronal cell death is significant at 1 week (McKay Hart et al. 2002) and known to involve selectively smaller neurons (Hu and McLachlan 2003; Tandrup et al. 2000). Therefore, a methodology to label the MG and sural neurons with Fast Blue whilst avoiding nerve transection was sought. Unfortunately, injection into the MG muscle or into the sural nerve labelled appreciably fewer neurons and may result in non-specific labelling via blood vessels (Perry and Lawson 1998); whilst the sural cutaneous region is inconstant and subject to innervation from neighbouring cutaneous nerves (Bajrovic and Sketelj 1998). These techniques may be acceptable when the principal aim is to sample a small number of neurons known to be uninjured; however, in this neuronal counting study it is arguable that the significantly smaller yield would bestow greater bias than peptide downregulation only a few days after injury. For these reasons it was decided to transect the nerves, apply the Fast Blue tracer in a consistent and established technique, and limit survival to 4 days: the earliest time-point at which the neuronal population was labelled with sufficient intensity, and cell death has not significantly established.

Another limitation of this study was the method of quantifying the immunohistochemistry. With approximately 3,500 neurons in the sural population and 220 in the MG population (Welin et al. 2008), there may have been a sample selection bias when restricting the sural neuron count to just over 200; however, this was limited by counting every fifth section, thereby ensuring that neurons were spread throughout the DRG. The phenotype staining of the MG and sural neurons was judged to be either positive or negative and immunohistochemistry staining intensity was not measured. Despite these limitations, the results showed that the phenotype proportions are consistent across three animals for each of MG and sural neuron counts. Furthermore, the effects of a sampling bias should be small when measuring the frequency of a chemically defined population that is 
co-staining another marker. Neuronal size measurements are also in keeping with previous reports (Swett et al. 1991).

Similarly in the gene expression analysis, a sample selection bias may be present as a result of capturing only 100 labelled sural or MG neurons via laser microdissection. Clearly, the sampled MG neurons amounting to more than $45 \%$ of the total population are likely to be better representation of the MG neuronal group than its sural counterpart which sampled only $3 \%$ of its total population. Given that there is no way of predicting which neurons will undergo apoptosis, the captured sural neurons also contain among them cells that will survive axotomy; however, with $15 \%$ undergoing apoptosis within the first week after injury and many more prepared to engage towards apoptosis in the weeks subsequent to this (McKay Hart et al. 2002), it was anticipated that a transcriptional response to the injury would be uncovered.

Cutaneous sensory afferents die in great numbers following axotomy, whereas muscular sensory afferents survive an identical insult ( $\mathrm{Hu}$ and McLachlan 2003; Welin et al. 2008). We utilised this model in conjunction with laser microdissection and qRT-PCR in order to highlight the differential transcriptional response of $\mathrm{MG}$ and sural neuron subpopulations following injury. Whilst activated caspase- 3 protein has been identified in morphologically apoptotic DRG neurons following distal axotomy (Kuo et al. 2005), our findings demonstrate that axotomised MG neurons significantly downregulate caspase-3 mRNA whereas the axotomised sural neurons significantly upregulate caspase- 3 transcripts at 1 week post-injury. This disparate response to injury is likely to be attributable to the differing IB4-positive composition of the subpopulations described. Previous studies have demonstrated that DRG IB4-positivity decreases following nerve injury (Bradbury et al. 1998; McMahon and Moore 1988); therefore, increasing caspase-3 gene expression in an axotomised sural subpopulation which is IB4-rich is in keeping with the current concept of sensory neuron response to injury. It is interesting that in response to injury MG neurons significantly downregulated caspase-3 mRNA to almost half of baseline levels which may indicate activation of a protective response, lacking in sural neurons. The underlying molecular mechanisms involved in axotomy-induced neuronal apoptosis have yet to be fully determined.

In conclusion, this study has demonstrated differing neurochemical phenotype and size characteristics which are likely to influence the apoptotic fates of sural and medial gastrocnemius DRG subpopulations. These findings provide further clarification in the understanding of two neuronal populations increasingly used in nerve injury models.
Acknowledgments We are grateful to D. Welin and L. Novikov for advice with the animal nerve injury model and retrograde fluorescent staining. We thank N. Gardiner for assistance with perfusion fixation techniques and the animal welfare workers at the University of Manchester for animal care. This work was funded by the Swedish Medical Research Council, Umeå University, County of Vasterbotten, Magn. Bergvalls Stiftelse, Gunvor and Josef Anér Foundation, the East Grinstead Medical Research Trust and the HB Allen Charitable Trust.

\section{References}

Ambalavanar R, Moritani M, Haines A, Hilton T, Dessem D (2003) Chemical phenotypes of muscle and cutaneous afferent neurons in the rat trigeminal ganglion. J Comp Neurol 460:167-179

Bajrovic F, Sketelj J (1998) Extent of nociceptive dermatomes in adult rats is not primarily maintained by axonal competition. Exp Neurol 150:115-121

Bergman E, Carlsson K, Liljeborg A, Manders E, Hokfelt T, Ulfhake B (1999) Neuropeptides, nitric oxide synthase and GAP-43 in B4-binding and RT97 immunoreactive primary sensory neurons: normal distribution pattern and changes after peripheral nerve transection and aging. Brain Res 832:63-83

Bradbury EJ, Burnstock G, McMahon SB (1998) The expression of P2X3 purinoreceptors in sensory neurons: effects of axotomy and glial-derived neurotrophic factor. Mol Cell Neurosci 12:256-268

Groves MJ, Christopherson T, Giometto B, Scaravilli F (1997) Axotomy-induced apoptosis in adult rat primary sensory neurons. J Neurocytol 26:615-624

Hammond DL, Ackerman L, Holdsworth R, Elzey B (2004) Effects of spinal nerve ligation on immunohistochemically identified neurons in the L4 and L5 dorsal root ganglia of the rat. J Comp Neurol 475:575-589

Hu P, McLachlan EM (2003) Selective reactions of cutaneous and muscle afferent neurons to peripheral nerve transection in rats. J Neurosci 23:10559-10567

Janicke RU, Sprengart ML, Wati MR, Porter AG (1998) Caspase-3 is required for DNA fragmentation and morphological changes associated with apoptosis. J Biol Chem 273:9357-9360

Jessell T, Tsunoo A, Kanazawa I, Otsuka M. (1979) Substance P: depletion in the dorsal horn of rat spinal cord after section of the peripheral processes of primary sensory neurons. Brain Res 168:247-259

Kuo LT, Simpson A, Schanzer A, Tse J, An SF, Scaravilli F, Groves MJ (2005) Effects of systemically administered NT-3 on sensory neuron loss and nestin expression following axotomy. J Comp Neurol 482:320-332

Lawson SN (2005) The peripheral sensory nervous system: dorsal root ganglion neurons. In: Dyck PJ, Thomas PK (eds) Peripheral Neuropathy. Saunders, Philadelphia, pp 163-185

Lawson SN, Crepps B, Perl ER (2002) Calcitonin gene-related peptide immunoreactivity and afferent receptive properties of dorsal root ganglion neurones in guinea-pigs. J Physiol 540:989-1002

McKay Hart A, Brannstrom T, Wiberg M, Terenghi G (2002) Primary sensory neurons and satellite cells after peripheral axotomy in the adult rat: timecourse of cell death and elimination. Exp Brain Res 142:308-318

McLachlan EM, Janig W (1983) The cell bodies of origin of sympathetic and sensory axons in some skin and muscle nerves of the cat hindlimb. J Comp Neurol 214:115-130

McMahon SB, Moore CE (1988) Plasticity of primary afferent acid phosphatase expression following rerouting of afferents from muscle to skin in the adult rat. J Comp Neurol 274:1-8

Molander C, Ygge J, Dalsgaard CJ (1987) Substance P-, somatostatinand calcitonin gene-related peptide-like immunoreactivity and 
fluoride resistant acid phosphatase-activity in relation to retrogradely labeled cutaneous, muscular and visceral primary sensory neurons in the rat. Neurosci Lett 74:37-42

Oaklander AL, Brown JM (2004) Unilateral nerve injury produces bilateral loss of distal innervation. Ann Neurol 55:639-644

O'Brien C, Woolf CJ, Fitzgerald M, Lindsay RM, Molander C (1989) Differences in the chemical expression of rat primary afferent neurons which innervate skin, muscle or joint. Neuroscience 32:493-502

Perry MJ, Lawson SN (1998) Differences in expression of oligosaccharides, neuropeptides, carbonic anhydrase and neurofilament in rat primary afferent neurons retrogradely labelled via skin, muscle or visceral nerves. Neuroscience 85:293-310
Puigdellivol-Sanchez A, Prats-Galino A, Molander C (2006) Estimations of topographically correct regeneration to nerve branches and skin after peripheral nerve injury and repair. Brain Res 1098:49-60

Swett JE, Torigoe Y, Elie VR, Bourassa CM, Miller PG (1991) Sensory neurons of the rat sciatic nerve. Exp Neurol 114:82-103

Tandrup T, Woolf CJ, Coggeshall RE (2000) Delayed loss of small dorsal root ganglion cells after transection of the rat sciatic nerve. J Comp Neurol 422:172-180

Welin D, Novikova LN, Wiberg M, Kellerth JO, Novikov LN (2008) Survival and regeneration of cutaneous and muscular afferent neurons after peripheral nerve injury in adult rats. Exp Brain Res $186: 315-323$ 